\title{
GENERIC HOMEOMORPHISMS HAVE NO SMALLEST ATTRACTORS
}

\author{
MIKE HURLEY \\ (Communicated by Charles C. Pugh)
}

\begin{abstract}
We show that if $M$ is a compact manifold then there is a residual subset $\mathscr{R}$ of the set of homeomorphisms on $M$ with the property that if $f \in \mathscr{R}$ then $f$ has no smallest attractor (that is, an attractor with the property that none of its proper subsets is also an attractor). Part of the motivation for this result comes from portions of a recent paper by Lewowicz and Tolosa that deal with properties of smallest attractors of generic homeomorphisms.
\end{abstract}

In this paper we consider the set of homeomorphisms of a compact manifold $M$ with metric $d$; this set of homeomorphisms is denoted as $\operatorname{Hom}(M)$ and is given the topology of uniform convergence, which makes $\operatorname{Hom}(M)$ a Baire space. An attractor for $f \in \operatorname{Hom}(M)$ is a compact, nonempty subset $A$ of $M$ with the following property: there is an open neighborhood $U$ of $A$ satisfying the two conditions (i) $f(\bar{U}) \subset U$ and (ii) $\cap_{n \geq 0} f^{n}(U)=A$. We will refer to such an open set $U$ as an attractor block that determines $A$ (this is slightly different from the standard definition of an attractor block). Note that $M$ itself is an attractor for any $f \in \operatorname{Hom}(M)$. The attractor $A$ is a smallest attractor for $f$ if no proper subset of $A$ is also an attractor for $f$; in other words, a smallest attractor is one that is minimal in the class of all attractors of $f$ when they are partially ordered by inclusion. The main result of this note is that there is a residual subset $\mathscr{R}$ of $\operatorname{Hom}(M)$ with the property that if $g \in \mathscr{R}$ then $g$ has no smallest attractor.

Remark 1. If $A$ is an attractor for $f$ and $W$ is any neighborhood of $A$, then there is an attractor block $U$ that determines $A$ with $A \subset U \subset W$; see [1,3].

There is a connection between the set of attractors of $f$ and its chain recurrent set. This connection was originally described by Conley [1] in the context of flows; for a development of this connection in the context of maps see [3] or [5] and the references there. For us the important result is that a point $x$ of $M$ is chain recurrent if and only if it has the following property: if some forward iterate of $x$ lies in an attracting block $U$ then $x$ lies in the attractor $A$ determined by $U$. In particular, an attractor $A$ for $f$ is a smallest attractor if and

Received by the editors July 1, 1993.

1991 Mathematics Subject Classification. Primary 58F12.

Key words and phrases. Attractor, generic homeomorphism. 
only if it is a chain transitive component of the chain recurrent set of $f$. This means that if $x$ and $y$ are any two points of $A$ and if $\epsilon>0$ then there is a finite sequence $x_{0}, x_{1}, \ldots, x_{n}$ with $x_{0}=x, x_{n}=y$, and $d\left(f\left(x_{j-1}\right), x_{j}\right)<\epsilon$ for each $j=1,2, \ldots, n$ (in the case $y=x$ we also require that $n \geq 1$ ). More generally, the chain recurrent set of $f$ is defined to be the set of all $x \in M$ with the property that for each $\epsilon>0$ there exist points $x_{0}, x_{1}, \ldots, x_{n}, n \geq 1$, with $x_{0}=x=x_{n}$ and $d\left(f\left(x_{j-1}\right), x_{j}\right)<\epsilon$ for each $j=1,2, \ldots, n$.

Generic properties of $\operatorname{Hom}(M)$ have been studied by several authors, notably $[8,2,7]$. Among the results that have been established are that there are residual subsets of $\operatorname{Hom}(M)$ satisfying each of the following properties.

Property G1. The periodic points of $f$ are dense in its chain recurrent set $[2,8]$; in particular periodic points are dense in any of its smallest attractors.

Property G2. $f$ has no periodic attractors [8].

In order to establish the generic nonexistence of smallest attractors, we will develop a third generic property, as follows. Suppose that $A$ is an attractor for $f$ with an attractor block $U$, so that $f(\bar{U}) \subset U$. Since $\bar{U}$ is compact, if $g \in \operatorname{Hom}(M)$ is sufficiently close to $f$ then $g(\bar{U}) \subset U$ as well, so that the set $A_{g}$ defined by $A_{g} \equiv \cap_{n \geq 0} g^{n}(U)$ is an attractor for $g$ contained in $U$. (In other words, an attractor block for $f$ is automatically an attractor block for all nearby $g \in \operatorname{Hom}(M)$.) Now suppose that $W$ is an arbitrary open subset of $M$. Define $\alpha_{W}(f) \in\{0,1,2, \ldots, \infty\}$ to be the largest number of pairwise disjoint attractors for $f$ that are contained in $W$. One can use Remark 1 to verify that if $A_{1}, \ldots, A_{k}$ are pairwise disjoint attractors for $f$ in $W$ then each $A_{j}$ has an attractor block $U_{j}$ with $U_{j}$ contained in $W$, and with $U_{1}, \ldots, U_{k}$ pairwise disjoint as well. This fact, coupled with the preceding observations, shows that the map $\alpha_{W}: M \rightarrow\{0,1,2, \ldots, \infty\}$ is lower semicontinuous (viewing $\{0,1,2, \ldots, \infty\}$ as the one-point compactification of $\{0,1,2, \ldots\}$ in the obvious way).

Lemma 1. For each nonempty open subset $W$ of $M$ there is a residual subset $C(W)$ of $\operatorname{Hom}(M)$ with the property that the map $\alpha_{W}: M \rightarrow\{0,1,2, \ldots, \infty\}$ defined above is continuous at each point of $C(W)$.

Proof. This follows from the discussion above, using the fact that the set of continuity points of a lower semicontinuous function forms a residual subset of its domain [9].

The rest of the argument is similar to [4]. Begin by selecting a countable basis $\mathscr{W}=\left\{W_{k} \mid k \geq 1\right\}$ for the topology of $M$, with the additional property that if $Y, Z$ are any disjoint closed subsets of $X$ then there are disjoint elements of $\mathscr{W}, W_{k}$ containing $Y$ and $W_{m}$ containing $Z$. (If $\mathscr{B}$ is an arbitrary countable basis for $M$ and $\mathscr{W}$ is composed of all finite unions of elements of $\mathscr{B}$, then $\mathscr{W}$ is a countable basis with this additional property.) Let $C \equiv \cap_{k \geq 1} C\left(W_{k}\right)$ where $C\left(W_{k}\right)$ is as in the lemma. Since the intersection is countable, $C$ is also a residual subset of $\operatorname{Hom}(M)$, and membership in $C$ defines our third generic property:

Property G3. Each map $\alpha_{W_{k}}$ is continuous at $f$. 
Theorem 2. Let $\mathscr{R} \subset \operatorname{Hom}(M)$ be the residual subset consisting of all homeomorphisms satisfying properties $\mathrm{G} 1, \mathrm{G} 2, \mathrm{G} 3$. If $f \in \mathscr{R}$ then $f$ has no smallest attractor.

Proof. We argue by contradiction; suppose that $f \in \mathscr{R}$ has a smallest attractor $A$. Let $U$ be an attractor block that determines $A$, and choose an element $W=W_{k}$ of the basis $\mathscr{W}$ with $A \subset W \subset U$. It follows that $\alpha_{W}(f)=1$. Since a smallest attractor is a subset of the chain recurrent set, $\mathrm{G} 1$ and $\mathrm{G} 2$ combine to show that $A$ contains two distinct periodic orbits, $f^{m}(p)=p$ and $f^{n}(q)=q$. If $\epsilon>0$ is given then there is a homeomorphism $g$, uniformly within $\epsilon$ of $f$, such that each of these two periodic orbits for $f$ is an attracting periodic orbit for $g$ (Lemma 3.1 of [8]). Clearly $\alpha_{W}(g) \geq 2$ for any such $g$, and since $\epsilon$ was arbitrary, this contradicts the continuity property $\mathrm{G} 3$.

Remark 2. Part of the motivation for this theorem comes from a recent paper by Lewowicz and Tolosa which, among other things, establishes properties of attractors for generic homeomorphisms (Proposition 2 and Theorem 2 of [6]). The attractors they consider are assumed to be either connected or topologically transitive subsets of the nonwandering set. The nonwandering set is contained in the chain recurrent set, and a connected or topologically transitive subset of the chain recurrent set is easily seen to be chain transitive, so the attractors considered in [6] are smallest attractors. Thus, unfortunately, those results concern smallest attractors of generic homeomorphisms, while the theorem above shows that, generically, homeomorphisms have no smallest attractors. In other words, their results deal with properties of smallest attractors for a residual set $\Sigma$ of homeomorphisms while the theorem shows that there is a residual subset $E$ of $\Sigma$ with the property that no $f$ in $E$ has any smallest attractors.

Remark 3. A little more work (along the lines of [4]) shows that a generic homeomorphism has no isolated chain transitive components. Here is a brief outline of the argument. For $\mathscr{W}=\left\{W_{k}\right\}$ as above, let $N_{k}(f)$ denoted the number of $W_{k}$ chain transitive components for $f$ (defined as in [4]). The first goal is to show that the set of continuity points for $N_{k}$ is residual in $\operatorname{Hom}(M)$. The key to the argument is to show that if $N_{k}(f)>0$ then there is an arbitrarily small perturbation $g$ of $f$ with the property that $N_{k}(h)>0$ for all $h$ in some neighborhood of $g$. Since (by property G1) we can assume that the periodic points of $f$ are dense in its chain recurrent set, we can again use Lemma 3.1 of [8] to perturb $f$ to $g$, changing a periodic orbit for $f$ into an attracting periodic orbit for $g$, so that, as above, any map $h$ close enough to $g$ will have a chain component in some neighborhood of the original periodic orbit. This gives a $C^{0}$ version of Lemma 7 of [4], from which the $C^{0}$ versions of Proposition 8 and Theorem 6 of [4] follow.

\section{ADDED IN PROOF}

It was pointed out by S. Pilyugin in his recent text (The space of dynamical systems with the $C^{0}$ topology, Lecture Notes in Math., vol. 1571, SpringerVerlag, Berlin and New York) that the arguments of [2] and [8] only show that property $\mathrm{G} 1$ is satisfied by elements of a residual subset of the $C^{0}$ closure of the $C^{1}$ diffeomorphisms. A proof of the genericity of $\mathrm{Gl}$ in the full set 
of homeomorphisms is contained in the preprint On proofs of the $C^{0}$ general density theorem by the author.

\section{REFERENCES}

1. C. Conley, Isolated invariant sets and the Morse index, Regional Conf. Ser. in Math., vol. 38, Amer. Math. Soc., Providence, RI, 1978.

2. E. M. Coven, J. Madden, and Z. Nitecki, A note on generic properties of continuous maps, Ergodic Theory and Dynamical Systems II, Birkhäuser, Boston, 1982, pp. 97-101.

3. R. Easton, Isolating blocks and epsilon chains for maps, Phys. D 39 (1989), 95-110.

4. M. Hurley, Bifurcation and chain recurrence, Ergodic Theory Dynamical Systems 3 (1983), 231-240.

5. _ Chain recurrence and attraction in non-compact spaces, Ergodic Theory Dynamical Systems 11 (1991), 709-729.

6. J. Lewowicz and J. Tolosa, On stable and unstable sets, Dynamical Systems (Santiago 1990), Pitman Res. Notes Math. Ser., vol. 285, Longman Sci. Tech., Harlow, 1993, pp. 117-130.

7. K. Odani, Generic homeomorphisms have the pseudo-orbit tracing property, Proc. Amer. Math. Soc. 110 (1990), 281-284.

8. J. Palis, C. Pugh, M. Shub, and D. Sullivan, Genericity theorems in topological dynamics, Dynamical Systems - Warwick 1974, Lecture Notes in Math., vol. 468, Springer-Verlag, New York, 1975, pp. 241-250.

9. F. Takens, On Zeeman's tolerance stability conjecture, Manifolds - Amsterdam, Lecture Notes in Math., vol. 197, Springer-Verlag, New York, 1971, pp. 209-219.

Department of Mathematics, Case Western Reserve University, Cleveland, Ohio 44106-7058

E-mail address: mgh3@po.cwru.edu 